\title{
Astragaloside IV alleviates heart failure by regulating SUMO-specific protease 1
}

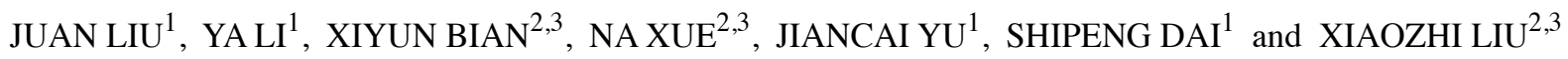 \\ ${ }^{1}$ Department of Cardiology, Cangzhou Central Hospital, Cangzhou, Hebei 061000; ${ }^{2}$ Central Laboratory, \\ ${ }^{3}$ Tianjin Key Laboratory of Epigenetics for Organ Development in Preterm Infants, \\ The Fifth Central Hospital of Tianjin, Tianjin 300450, P.R. China
}

Received September 21, 2020; Accepted May 13, 2021

DOI: $10.3892 /$ etm.2021.10510

\begin{abstract}
The present study investigated whether the protective effect and mechanism of astragaloside IV (AS-IV) on heart failure (HF) involves small ubiquitin-like modifier (SUMO)-specific protease 1 (Senp1). Mouse HF was established by aortic constriction, inducing pressure overload. The model was confirmed by echocardiography 6 weeks after surgery. Mice were randomly divided into control, HF, $\mathrm{HF}+\mathrm{AS}-\mathrm{IV}$, and AS-IV groups. Ventricular function was examined by echocardiography. Morphological changes of myocardial tissues were examined by H\&E staining. The protein levels of the apoptosis-related proteins, cleaved caspase-3, caspase-3, Bcl2, Bax, and SUMO-Senp1 were determined by Western blotting. $\mathrm{H}_{2} \mathrm{O}_{2}$ in isolated mitochondria and cells was determined by Amplex Red. A reactive oxygen species (ROS) detection kit determined ROS levels in isolated mitochondria and HL-1 cells. JC-1 reagent measured mitochondrial membrane potential $(\Delta \Psi \mathrm{m})$. Apoptosis of HL-1 cells was examined by terminal deoxynucleotidyl transferase dUTP nick end labeling. Compared with the control group, the heart weight and heart mass/body weight ratio increased in the HF group $(\mathrm{P}<0.05)$. Furthermore, the ejection fraction and left ventricular shortening fraction decreased $(\mathrm{P}<0.05)$, while the left ventricular end-diastolic diameter (LVID;d) and end-systolic diameter (LVID;s) increased $(\mathrm{P}<0.05)$. Finally, mitochondrial ROS and $\mathrm{H}_{2} \mathrm{O}_{2}$ increased $(\mathrm{P}<0.05)$, while the $\Delta \Psi \mathrm{m}$ decreased $(\mathrm{P}<0.05)$. However, AS-IV improved the
\end{abstract}

Correspondence to: Dr Xiaozhi Liu, Tianjin Key Laboratory of Epigenetics for Organ Development in Preterm Infants, The Fifth Central Hospital of Tianjin, 41 Zhejiang Road, Tanggu Street, Binhai New, Tianjin 300450, P.R. China

E-mail: 1xz7997@126.com

Dr Shipeng Dai, Department of Cardiology, Cangzhou Central Hospital, 16 Xinhua West Road, Yunhe, Cangzhou, Hebei 061000, P.R. China

E-mail: czyjc@163.com

Key words: astragaloside IV, heart failure, SUMO protease 1, oxidative stress, mitochondrial membrane potential cardiac function of HF mice, decreased the level of ROS and $\mathrm{H}_{2} \mathrm{O}_{2}$ in the myocardium, suppressed the decrease in $\Delta \Psi \mathrm{m}$, and decreased the apoptosis of myocardial cells $(\mathrm{P}<0.05)$. AS-IV also decreased the Senp1-overexpression. Furthermore, in HL-1 cells, Senp1-overexpression significantly inhibited the protective effects of AS-IV. AS-IV decreased oxidative stress in cardiomyocytes, decreased mitochondrial damage, inhibited ventricular remodeling, and ultimately improved cardiac function by inhibiting HF-induced Senp1-overexpression. This mechanism provides a novel theoretical basis and clinical treatment for HF.

\section{Introduction}

Chronic heart failure (CHF) is a worldwide public health problem that severely threatens human health. Its main manifestation is abnormal cardiac structure, leading to impaired cardiac filling or ejection function (1). The majority of cases of heart failure (HF) have a poor prognosis. According to European data, acute HF accounts for $17.4 \%$ of all-cause mortality per year, while CHF accounts for $7.2 \%$ (2). In high-income countries, the cost of HF treatment accounts for $2-3 \%$ of the total health system expenditure, and is expected to more than double in the next 20 years (3). At present, the clinical treatment of HF drugs mainly includes diuretics, angiotensin converting enzyme inhibitors, angiotensin receptor blockers and glucocorticoid receptor antagonists (4). Although there are diverse HF clinical treatments, the mortality and disability rates due to $\mathrm{HF}$ remain high (5).

Traditional Chinese Medicine, including astragalus, ginseng, ginsenoside and pepperweed seed, has a long history in the treatment of HF, and has the advantages of multiple targets, low cost and few side effects (6). Astragalus has been widely used in the treatment of cardiovascular diseases in China, and astragaloside IV (AS-IV) is one of its important effective components. The main pharmacological effects of AS-IV include enhancing immunity, anti-inflammatory, antioxidation and anti-viral (7). In recent years, it has been demonstrated that AS-IV exerts cardiac protection by regulating intracellular calcium homeostasis and the antioxidant response, improving myocardial energy metabolism, inhibiting apoptosis and alleviating cytotoxicity. The results of a previous study supported the effectiveness and safety of AS-IV in HF 
models in vivo and in vitro (7). However, the mechanism of its action in $\mathrm{HF}$ requires further study.

Ubiquitin is a small molecule protein that exists in the majority of eukaryotic cells. The main function of protein ubiquitination is to mark the proteins for degradation, followed by removal by proteolytic enzyme hydrolysis. More than $80 \%$ of cellular proteins are degraded by the ubiquitin proteasome system (8). Small ubiquitin-like modifier (SUMO) is a post-translational modification protein with similar structure but different functions to ubiquitin. SUMO binds to specific lysine sites on the target protein, to stabilize the target protein structure, mediate cytosol-nuclear translocation, regulate downstream transcription factors, regulate protein interactions and suppress ubiquitination (9). Like the ubiquitination pathway, SUMO precursor synthesis, hydrolysis and activation involve a series of enzymes, including E1 activating enzyme, E2 binding enzyme, E3 ligase and SUMO proteases (SENPs). At present, six SENP proteins (SENP1, SENP2, SENP3, SENP5, SENP6, SENP7) participate in the SUMOylation process. Furthermore, deSUMOylation serves an important role in determining the degree of protein SUMOylation (10). It has been demonstrated that SENP1, an important deSUMOylation enzyme, serves a central role in inhibiting cardiac hypertrophy and cardiac dysfunction (11). It has also been demonstrated that SENP1 serves an important regulatory role in oxidative stress and mitochondrial damage (12). However, its role in the AS-IV protective effect has not been reported. Therefore, it was unclear whether AS-IV inhibits the occurrence and development of HF by regulating SENP1.

In the present study, a mouse HF model was established by aortic coarctation, and the protective effects of AS-IV on cardiac function and oxidative stress of myocardial cells were examined in vivo. Furthermore, the expression of Senp1 in the HL-1 cell line was modulated to determine whether the protective effects of AS-IV on HF myocardium are associated with Senp1. The results of the present study provided a novel theoretical basis and clinical reference for HF treatment based on SUMOylation in the future.

\section{Materials and methods}

Animals and main reagents. Male C57BL/6J mice (8 weeks old) were purchased from the Institute of Experimental Animals, Chinese Academy of Medical Sciences. The mouse atrial muscle HL-1 cell line was purchased from ATCC. A lentiviral plasmid containing HA-Senpl was constructed by Suzhou Jima gene Co., Ltd. AS-IV was purchased from Sigma-Aldrich; Merck KGaA. Claycomb Medium (51800C), penicillin/streptomycin (V900929) and glutamine (200 mM, G8540) were from Sigma-Aldrich; Merck KGaA. Fetal bovine serum (10099141C) and trypsin (25200072) were purchased from Gibco; Thermo Fisher Scientific, Inc. Antibodies against cleaved-caspase-3 (cat. no. 9661, 1:1,000), caspase-3 (cat. no. 9662, 1:1,000), BCL2 (cat. no. 15071, 1:1,000), Bax (cat. no. 2774, 1:1,000), Senp1 (cat. no. 11929, 1:1,000) and $\beta$-actin (cat. no. 3700, 1:1,000) were purchased from Cell Signaling Technology, Inc. Goat anti-mouse IgG (cat. no. 115-035-003) and goat anti-rabbit IgG (cat. no. 111-035-003) were purchased from Jackson Laboratory. Amplex Red reagent (cat. no. A22177) was purchased from Invitrogen; Thermo Fisher Scientific, Inc. Mitochondrial membrane potential detection kit (kit. no. C2006), lactate dehydrogenase (LDH) cytotoxicity test kit (kit. no. C0016), and reactive oxygen species (ROS) detection kit (kit. no. S0033M) were purchased from Shanghai Beyotime Biotechnology Co., Ltd. Terminal deoxynucleotidyl transferase dUTP nick end labeling (TUNEL) kit (kit. no. 11684817910) was purchased from Roche Diagnostics GmbH.

Animal model. Animal experiments were performed according to the regulations and guidelines approved by the Animal Ethics Committee of The Fifth Central Hospital of Tianjin (approval. no. TJWZX2019018). Animal studies adhered to the Guide for the Care and Use of Laboratory Animals (8th edition, 2011, the Institute for Laboratory Animal Research of the National Research Council in the USA). A total of 24 male C57BL/6J mice (8-week-old; mean weight, $20 \mathrm{~g}$ ) were purchased from the Animal Center of Nanjing University. Animals were housed in the Experimental Animal Center of The Fifth Central Hospital of Tianjin, and maintained under a controlled temperature $\left(22-24^{\circ} \mathrm{C}\right)$, stable humidity (40-60\%) and a $12 \mathrm{~h}$-light/dark cycle with ad libitum access to food and water. To establish the HF model, the mice were anesthetized with 2\% isoflurane from Zaozhuang Shuitailan Chemical Co. Ltd., and then transferred to the operating table. Following endotracheal intubation, they were connected to a ventilator, and further anesthetized with $1.5 \%$ isoflurane. After the mouse chest was disinfected, a longitudinal incision was made in the second rib to open the chest and expose the thoracic segment of the aorta. Finally, a 7-0 thread was inserted below the brachiocephalic trunk of the aorta and the left common carotid artery. A $26 \mathrm{G}$ fine needle was used to ligate the aortic arch, and the needle was removed following ligation. After 6 weeks, echocardiography was used to determine whether the model was successfully established. The mice were randomly divided into control group, HF group, HF+AS-IV group and AS-IV group. The HF+AS-IV group and AS-IV group were intraperitoneally injected with $40 \mathrm{mg} / \mathrm{kg}$ AS-IV every other day. The control group and HF group were given the same dose of solvent (dimethyl sulfoxide, $0.1 \%$ ). After 4 weeks of drug intervention, the mice underwent cardiac ultrasound examination. At the end of the experiments, the mice were euthanized by $\mathrm{CO}_{2}$ exposure $\left(\mathrm{CO}_{2}\right.$ displacement rate equivalent to $20 \%$ of the chamber volume/min, October 2019) and cervical dislocation.

Cell culture. HL-1 cardiomyocytes from mouse heart tissue were purchased from ATCC. The cells were cultured in Dulbecco's modified Eagle's medium (Invitrogen; Thermo Fisher Scientific, Inc.) supplemented with $10 \%$ fetal bovine serum, $100 \mathrm{U}$ penicillin/streptomycin and $2 \mathrm{mM}$ glutamine at $37^{\circ} \mathrm{C}, 5 \% \mathrm{CO}_{2}$, 95\% air and $95 \%$ humidity. Following vector or HA-Senpl plasmid transfection for $24 \mathrm{~h}$, the HF group was treated with isoprenaline (ISO, $20 \mu \mathrm{mol} / \mathrm{l}$ ) for $24 \mathrm{~h}$, and the HF+AS-IV group was pretreated with $25,50,100$ or $200 \mu \mathrm{mol} / 1 \mathrm{AS}-\mathrm{IV}$ for $30 \mathrm{~min}$ at $37^{\circ} \mathrm{C}$, and then with $20 \mu \mathrm{mol} / 1 \mathrm{ISO}$ for $24 \mathrm{~h}$ at $37^{\circ} \mathrm{C}$. The mitochondrial membrane potential $(\Delta \psi \mathrm{m})$ and ROS were examined after $24 \mathrm{~h}$ of treatment.

Isolation of mouse left ventricular mitochondria. The mitochondria and cytoplasm were separated by a Tissue 
Mitochondrial Separation kit (Beyotime Institute of Biotechnology) using differential centrifugation, according to the manufacturer's protocols. Briefly, heart tissue blocks of the same weight and from the same region were washed with precooled PBS solution, cut into small pieces and transferred to a precooled glass homogenizer. Mitochondrial isolation solution containing protease inhibitors was added and the tissue was homogenized in an ice bath $\sim 10$ times. The supernatant was extracted following centrifugation at $4^{\circ} \mathrm{C}$ for $5 \mathrm{~min}$ at $600 \mathrm{x} \mathrm{g}$. Subsequently, the supernatant was discarded following centrifugation at $4^{\circ} \mathrm{C}$ for $10 \mathrm{~min}$ at $11,000 \mathrm{x} \mathrm{g}$. The remaining precipitate comprised the isolated mitochondria.

Measurement of mitochondrial membrane potential. Isolated mitochondria or HL-1 cells were incubated with JC-1 $\left(10 \mu \mathrm{g} / \mathrm{ml}\right.$ ) for $20 \mathrm{~min}$ at $37^{\circ} \mathrm{C}$. Mitochondria were examined by a fluorescence reader and HL-1 cells were examined by a laser scanning confocal microscope (Olympus FV 1200; Olympus Corporation; magnification $\mathrm{x} 60$ ). The excitation and emission wavelengths of the green fluorescence of JC-1 monomer were 488 and $525 \mathrm{~nm}$, respectively, and of the red fluorescence of JC-1 aggregate were 543 and $590 \mathrm{~nm}$, respectively. The change in fluorescence intensity of each experimental group is expressed as polymer/monomer. The temperature was maintained at $37^{\circ} \mathrm{C}$.

Measurement of ROS. Isolated myocardial mitochondria or HL-1 cells were incubated with DCFH-DA $(10 \mu \mathrm{M})$ for $20 \mathrm{~min}$ at $37^{\circ} \mathrm{C}$. Mitochondria were examined by a fluorescence reader and HL-1 cells were examined by a laser scanning confocal microscope (Olympus FV 1200; Olympus Corporation; magnification $\mathrm{x} 100)$. The excitation and emission wavelengths of DCFH-DA were 488 and $525 \mathrm{~nm}$, respectively. The fluorescence intensity of DCFH-DA is expressed as a percentage of the control group.

Measurements of mitochondrial hydrogen peroxide $\left(\mathrm{H}_{2} \mathrm{O}_{2}\right)$. The Amplex Red $\mathrm{H}_{2} \mathrm{O}_{2}$ detection kit was used according to the manufacturer's protocols; $\mathrm{H}_{2} \mathrm{O}_{2}$ in isolated mitochondria or HL-1 cells was detected by a fluorescence plate reader. The excitation and emission wavelengths of $\mathrm{H}_{2} \mathrm{O}_{2}$ were 543 and $590 \mathrm{~nm}$, respectively. The change in fluorescence intensity in each experimental group is expressed as a percentage of the control group.

Western blotting. Total protein was extracted from cells or tissues using RIPA buffer (Beijing Solarbio Science \& Technology Co., Ltd.) supplemented with $1 \mathrm{mM}$ PMSF and $20 \mathrm{mM}$ N-ethylmaleimide. The protein supernatant was collected following centrifugation at $10,000 \mathrm{x} \mathrm{g}$ at $4^{\circ} \mathrm{C}$ for $15 \mathrm{~min}$. Protein concentration was measured using a BCA protein assay (Beijing Solarbio Science \& Technology Co., Ltd.). Equal amounts of protein lysates ( $40 \mu \mathrm{g}$ per lane; $2 \mu \mathrm{g} / \mu \mathrm{l})$ were separated on SDS-polyacrylamide gel using $10 \%$ gels and were transferred onto PVDF membranes. The membranes were then blocked with $5 \%$ skimmed milk for $1 \mathrm{~h}$ at room temperature. Next, the membranes were incubated overnight with the aforementioned primary antibodies at $4^{\circ} \mathrm{C}$ overnight, and then with secondary antibodies for $1 \mathrm{~h}$ at room temperature. Finally, the protein bands were visualized by enhanced chemiluminescence (EMD Millipore). Densitometric semi-quantification analysis of the Western blot bands was performed using image analysis software (ImageJv1.48; National Institutes of Health). Protein band intensity was normalized to $\beta$-actin and expressed as a percentage of the naive control.

TUNEL staining. To detect apoptosis, a TUNEL kit (Roche Diagnostics $\mathrm{GmbH}$ ) was used. Following treatment, HL-1 cells were fixed in $4 \%$ paraformaldehyde for $40 \mathrm{~min}$ at $25^{\circ} \mathrm{C}$, blocked with $3 \% \mathrm{H}_{2} \mathrm{O}_{2}$ for $10 \mathrm{~min}$ at $25^{\circ} \mathrm{C}$, and permeated with $0.1 \%$ Triton X-100 for $3 \mathrm{~min}$. Apoptotic cells were labeled with the TUNEL reaction mixture and nuclei were stained with DAPI $(1 \mu \mathrm{g} / \mathrm{ml})$ for $3 \mathrm{~min}$ at $25^{\circ} \mathrm{C}$. Cells were washed twice with PBS for $5 \mathrm{~min}$ at room temperature and mounted with ProLong Gold Antifade reagent (Invitrogen; Thermo Fisher Scientific, Inc.). Images were randomly obtained using a fluorescence microscope (Olympus ix73; Olympus Corporation; dp73 camera; magnification x10).

Echocardiography. Eight weeks after the operation, mice were anesthetized with pentobarbital sodium $(30 \mathrm{mg} / \mathrm{kg})$ and placed on a warm pad. Vevo $770^{\circledR}$ (Visualsonics Inc.) and a 716 probe were used to dynamically evaluate the cardiac function of mice by echocardiography. Left ventricular end-systolic diameter (LVESD) and left ventricular end-diastolic diameter (LVEED) were obtained by M-type tracing. Ejection fraction (EF) and shortening fraction (FS) were automatically obtained by high-resolution echocardiography (ECG).

Histological examination. Myocardial tissue was fixed with $4 \%$ paraformaldehyde for $24 \mathrm{~h}$ at $4^{\circ} \mathrm{C}$, dehydrated, paraffin embedded and sectioned ( $4 \mu \mathrm{m}$ thick). Pathological sections were stained with hematoxylin for $5 \mathrm{~min}$ at $4^{\circ} \mathrm{C}$ and eosin for $3 \mathrm{~min}$ at $4^{\circ} \mathrm{C}$ (Nanjing SenBeiJia Biological Technology Co., Ltd.). H\&E staining was observed under a light microscope (Olympus Medical Systems; magnification, x20). The hypertrophy of cardiomyocytes was quantified by counting the cross-sectional area of cardiomyocytes using Image-Pro Plus 6.0 (Media Cybernetics, Inc.).

HA-Senpl-overexpression plasmid and cell transfection. Total RNA was extracted from the HL-1 cells using a TRIzol ${ }^{\circledR}$ RNA extraction kit (Tiangen Biotech Co., Ltd.). The RNA extracted from HL-1 cells was used to obtain the full-length cDNA of mouse Senpl by RT-PCR using a RT-PCR kit according to the manufacturer's protocol (Thermo Fisher Scientific, Inc.). The full-length Senpl cDNA (Accessions; NM_001379573.1; Insert size: 2019 bp) was subcloned into mammalian Lentivirus Expression Vector pwpxl (Thermo Fisher Scientific, Inc.) with a HA tag at the C-terminus (HA-SENP1), and the empty plasmid, pwpxl, was used as the negative control group (vector). The empty vector and the vectors encoding the SENP1 (HA-SENP1) were transiently transfected into HL-1 cells using Lipofectamine 2000 (Invitrogen; Thermo Fisher Scientific, Inc.) according to the manufacturer's protocols (DNA $=4 \mu \mathrm{g}$, reagent $=10 \mu \mathrm{l}$ ). In brief, cells were seeded into a 6-well plate at $37^{\circ} \mathrm{C}, 5 \% \mathrm{CO}_{2}$ and $95 \%$ humidity. A total of 18-24 h after seeding, the density of the HL-1 cells reached $40-50 \%$, prior to being transfected with empty vector or 
A

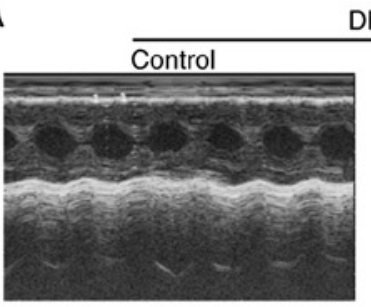

DMSO
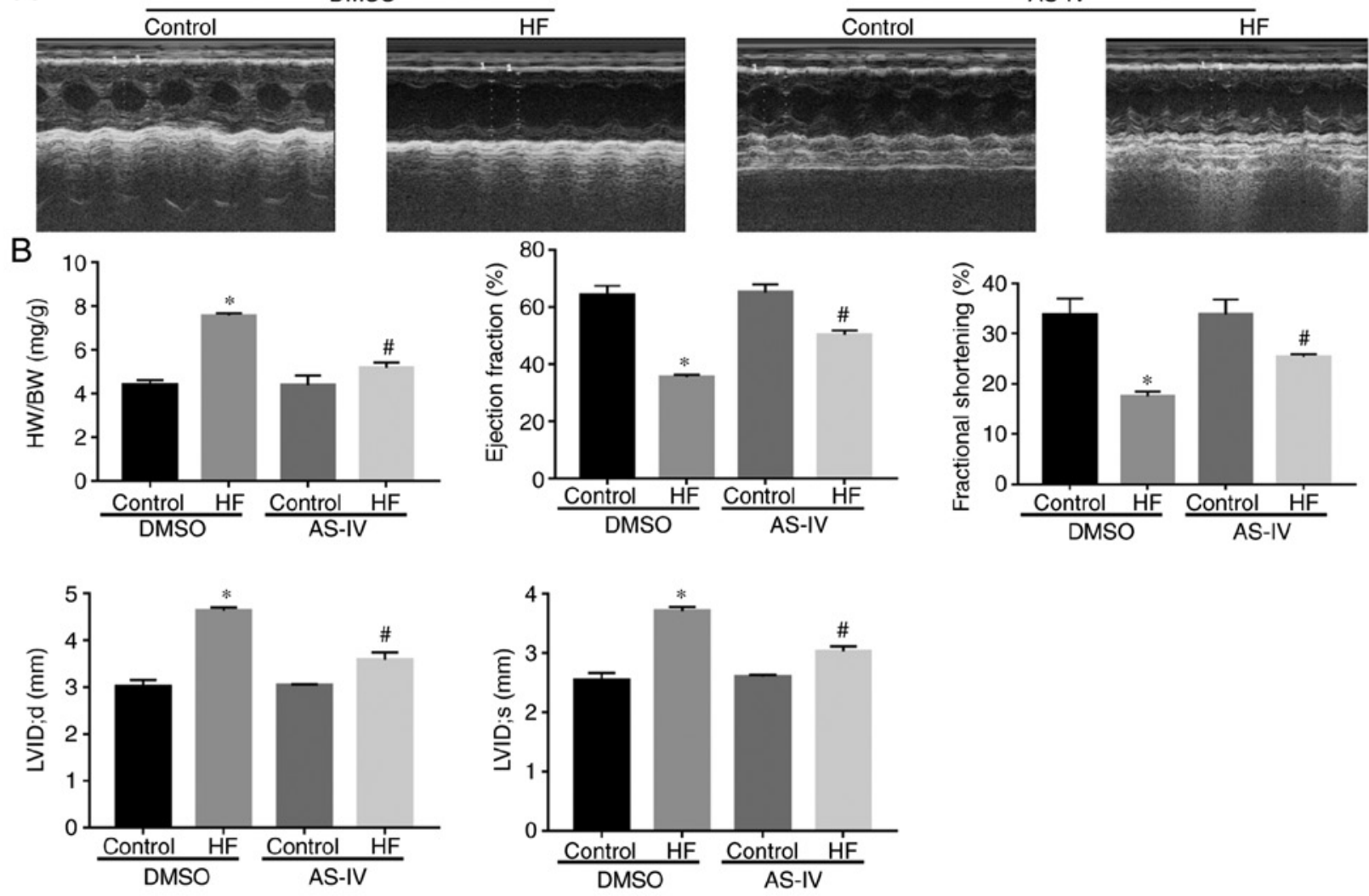

Figure 1. AS-IV treatment improved the contraction function in transverse aortic constriction-induced HF. (A) Representative M-mode echocardiography images. (B) Summarized data of HW/BW, ejection fraction, fractional shortening; LVIDs, LVIDd, $(\mathrm{n}=6)$. Data are presented as the mean \pm standard deviation. One-way analysis of variance, followed by Tukey's test. ${ }^{*} \mathrm{P}<0.05$ vs. control; ${ }^{\sharp} \mathrm{P}<0.05$ vs. HF. AS-IV, astragaloside; HF, heart failure; HW/BW, heart mass/body weight; EF, ejection fraction; FS, fractional shortening; LVIDs, left ventricular internal systolic diameter; LVIDd, left ventricular internal diastolic diameter; DMSO, dimethyl sulfoxide.

HA-SENP1 using Lipofectamine 2000 transfection reagent (DNA $=4 \mu \mathrm{g}$, reagent $=10 \mu \mathrm{l}$ ). Cells were then inoculated into fresh medium $24 \mathrm{~h}$ after the transfection. All subsequent experiments were performed $48 \mathrm{~h}$ after transfection.

Cell viability assay. Cell viability was examined using a Cell Counting Kit-8 (CCK-8; Beijing Solarbio Science \& Technology Co., Ltd.) assay. H1-1 cells were cultured in 96-well plates $\left(2 \times 10^{3}\right.$ cells/well $)$ and treated as described in 'cell culture'. Subsequently, $10 \mu \mathrm{l}$ CCK-8 solution was added to each well and incubated at $37^{\circ} \mathrm{C}$ for $2 \mathrm{~h}$. A microplate reader (VersaMax $^{\mathrm{TM}}$ Microplate Reader; Molecular Devices, LLC) was used to analyze the absorbance at $450 \mathrm{~nm}$. To increase the reliability of measures, each experiment was performed three times.

Statistical analysis. All HL-1 cell culture dishes and mice were randomly assigned to different experimental groups. Data are presented as the mean \pm standard deviation. Statistical analysis was performed using GraphPad Prism 6.0 software (GraphPad Software, Inc.). The differences between the experimental and control groups were tested using unpaired t-test. For comparing differences among more than two experimental groups, the means were compared using one-way analysis of variance (ANOVA), followed by Tukey's test, or two-way ANOVA, followed by Bonferroni's test. Normal distribution was assessed with the Shapiro-Wilk test.
$\mathrm{P}<0.05$ was considered to indicate a statistically significant difference.

\section{Results}

$A S-I V$ treatment improves contraction function in transverse aortic constriction (TAC)-induced heart failure. Compared with the control group, the heart weight and the heart mass/body weight ratio were increased in the HF group $(\mathrm{P}<0.05)$; however, AS-IV reversed this increase induced by pressure overload-driven heart failure $(\mathrm{P}<0.05$; Fig. 1$)$. The ultrasound results demonstrated that, compared with the control group, the EF, FS, LVID;d and LVID;s were increased in the HF group $(\mathrm{P}<0.05$; Fig. $1 \mathrm{~A}$ and $\mathrm{B})$, suggesting that the heart function of the HF group was impaired. However, AS-IV improved the heart function.

AS-IV reduces TAC-induced cardiomyocyte hypertrophy and mitochondrial damage. H\&E staining demonstrated that, compared with the control group, myocardial cells in the HF group exhibited hypertrophy, disordered arrangement, increased intercellular stroma and inflammatory infiltration. However, AS-IV reversed the morphological changes of the myocardial tissue induced by TAC (Fig. 2). Furthermore, compared with the control group, the ROS levels in myocardial mitochondria of the HF group were increased, while AS-IV decreased the ROS content in HF myocardial cells (Fig. 3A). 
A

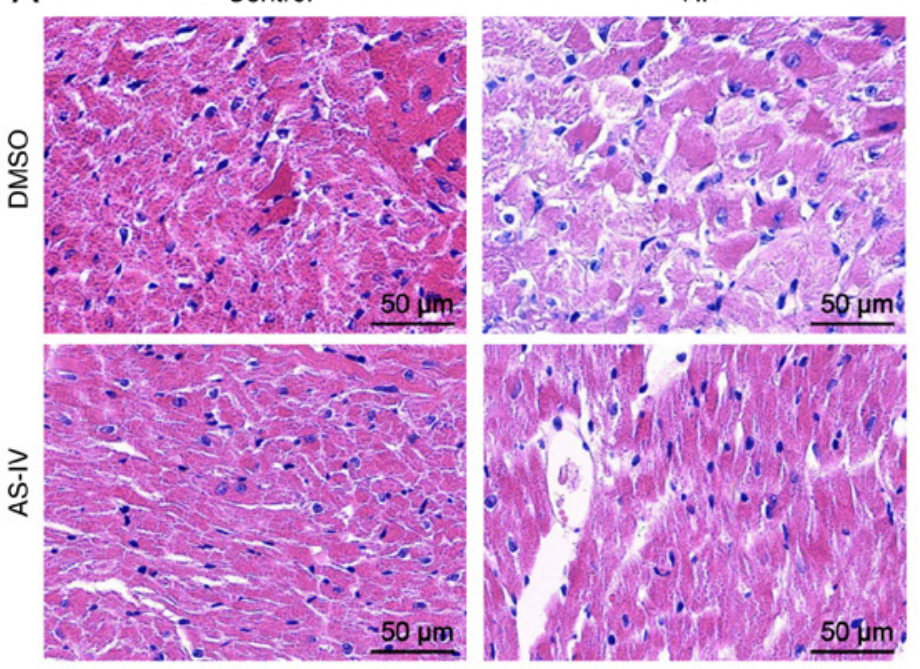

B

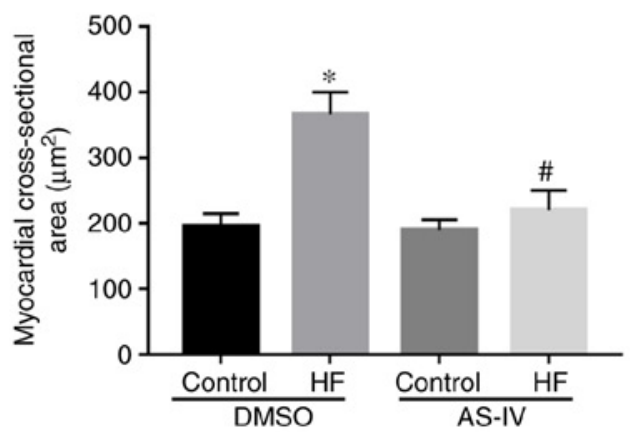

Figure 2. H\&E staining showing the protective effect of AS-IV on transverse aortic constriction-induced HF. (A) Myocardial cells showed hypertrophy, disordered arrangement, increased intercellular stroma and inflammatory infiltration in the HF group, which were reversed by AS-IV. (B) Summarized data of H\&E staining $(n=6)$. Data are presented as the mean \pm standard deviation. One-way analysis of variance, followed by Tukey's test. * $\mathrm{P}<0.05$ vs. control; ${ }^{\#} \mathrm{P}<0.05$ vs. HF. AS-IV, astragaloside; HF, heart failure; DMSO, dimethyl sulfoxide.
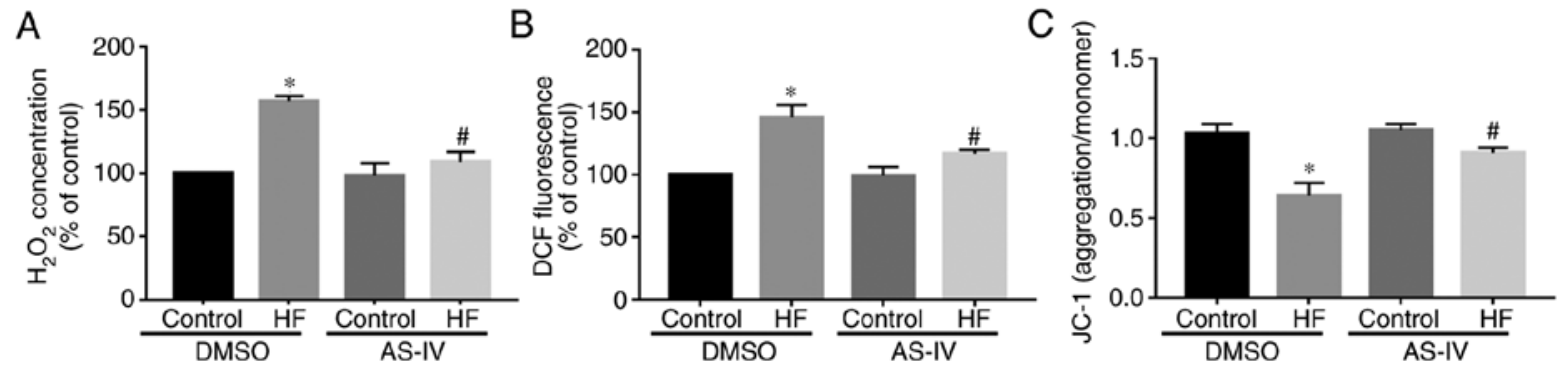

Figure 3. Effects of AS-IV on oxidative stress and mitochondrial membrane potential in transverse aortic constriction-induced heart failure. (A) ROS levels were tested by DCFH-DA. (B) $\mathrm{H}_{2} \mathrm{O}_{2}$ was tested by an Amplex Red kit ( $\mathrm{n}=6$ ). (C) The mitochondrial membrane potential was tested by JC-1 ( $=6$ ). Data are presented as the mean \pm standard deviation. One-way analysis of variance, followed by Tukey's test. ${ }^{*} \mathrm{P}<0.05$ vs. control; ${ }^{\#} \mathrm{P}<0.05$ vs. HF. AS-IV, astragaloside; $\mathrm{HF}$, heart failure; $\mathrm{H}_{2} \mathrm{O}_{2}$, hydrogen peroxide; DMSO, dimethyl sulfoxide.

The Amplex Red results demonstrated that, compared with the control group, the $\mathrm{H}_{2} \mathrm{O}_{2}$ content in myocardial mitochondria of the HF group was increased, which was abrogated by AS-IV (Fig. 3B). The JC-1 results demonstrated that, compared with the control group, the $\Delta \psi \mathrm{m}$ of the HF group was decreased, which was also reversed by AS-IV (Fig. 3C).

Expression of cardiomyocyte apoptosis-related proteins and Senpl protein. Western blotting demonstrated that, compared with the control group, the cleaved-caspase-3/caspase-3 ratio was increased, but the $\mathrm{Bcl} 2 / \mathrm{Bax}$ ratio was decreased in the HF group, indicating that apoptosis of cardiomyocytes in the HF group was increased. This effect was abolished by AS-IV (Fig. 4A and B). Additionally, compared with the control group, the expression of Senp1 was increased in the HF model, while AS-IV decreased this expression (Fig. 4A and B), indicating that Senp1 may participate in the myocardial protection of AS-IV.

Effect of Senpl-overexpression on oxidative stress. To further investigate the effect of Senp1-overexpression on oxidative stress in HL-1 cells, the present study examined the effect of different concentrations of AS-IV on the survival of HL-1 cells subjected to ISO, which induces myocardial injury. The CCK-8 results demonstrated that HL-1 cells treated with ISO had significantly decreased cell viability. AS-IV at 50 and $100 \mathrm{mmol} / \mathrm{l}$ significantly improved the cell viability, but the protective effect of 25 and $200 \mathrm{mmol} / \mathrm{l}$ was not notable (Fig. 5A). Next, the HA-Senp1 plasmid was transfected into HL-1 cells. In the subsequent experiments, $50 \mathrm{mmol} / \mathrm{l} \mathrm{AS-IV} \mathrm{was} \mathrm{used} \mathrm{as} \mathrm{the}$ intervention concentration. Western blotting demonstrated that, compared with the vector group, the expression of Senp1 was increased in the HA-Senpl group (Fig. 5B). Compared with the $\mathrm{HF}$ group, AS-IV decreased the increase in intracellular $\mathrm{H}_{2} \mathrm{O}_{2}$, while the Senp1-overexpression in HL-1 cells inhibited the effect of AS-IV (Fig. 5C). Furthermore, confocal detection of DCF demonstrated that AS-IV decreased the increase in ROS compared with the HF group, while Senp1-overexpression in HL-1 cells abolished the effect of AS-IV (Fig. 6).

Effects of Senpl-overexpression on $\Delta \psi m$ and apoptosis of $H L-1$ cells. Mitochondria are associated with apoptosis, and the decline in $\Delta \psi \mathrm{m}$ is an important indicator of early apoptosis. Confocal detection of JC-1 demonstrated that, compared 


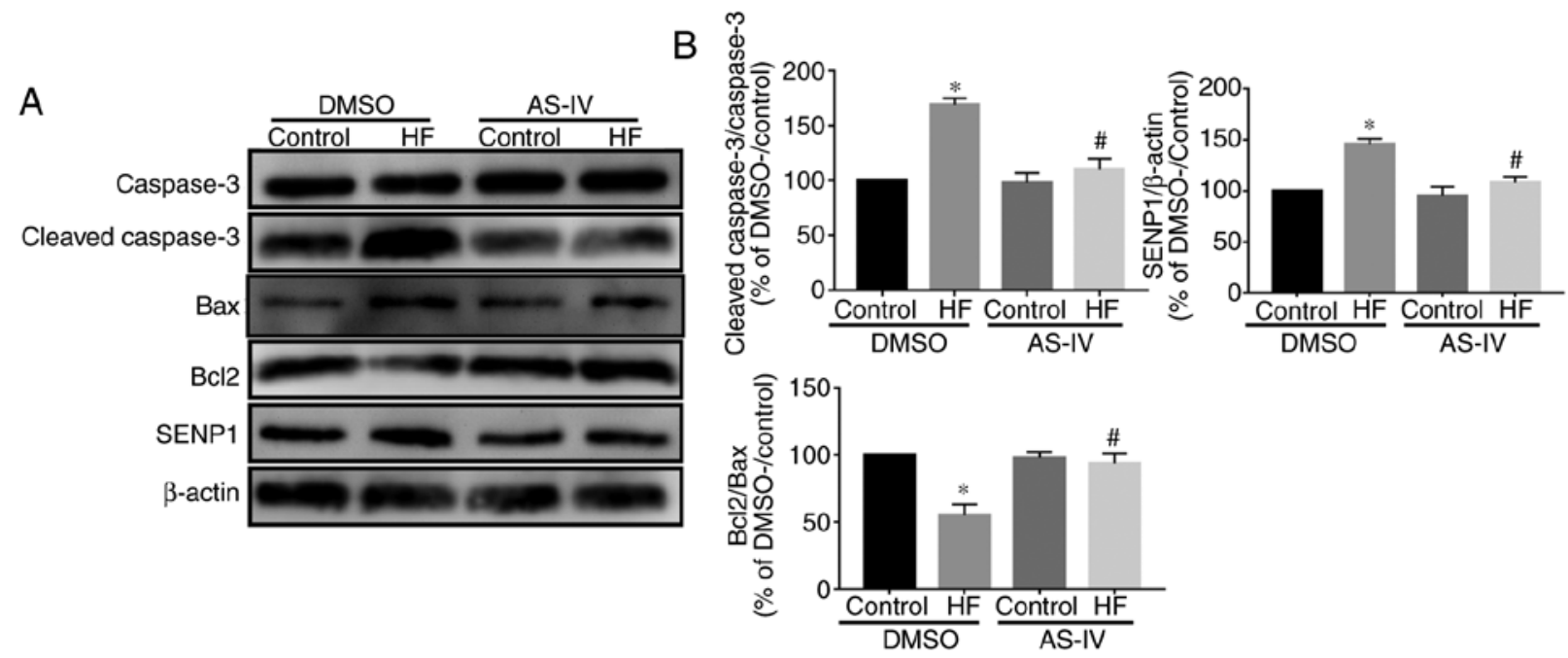

Figure 4. AS-IV reversed the transverse aortic constriction-induced expression of apoptosis-related proteins and Senp1. (A) Apoptosis-related proteins and Senp1 expression was examined by Western blotting. (B) Quantification of the results in A $(n=6)$. Data are presented as the mean \pm standard deviation. One-way analysis of variance, followed by Tukey's test. "P $<0.05$ vs. control; ${ }^{\#} \mathrm{P}<0.05$ vs. HF. AS-IV, astragaloside; Senp1, small ubiquitin-like modifier-specific protease 1; HF, heart failure; DMSO, dimethyl sulfoxide.
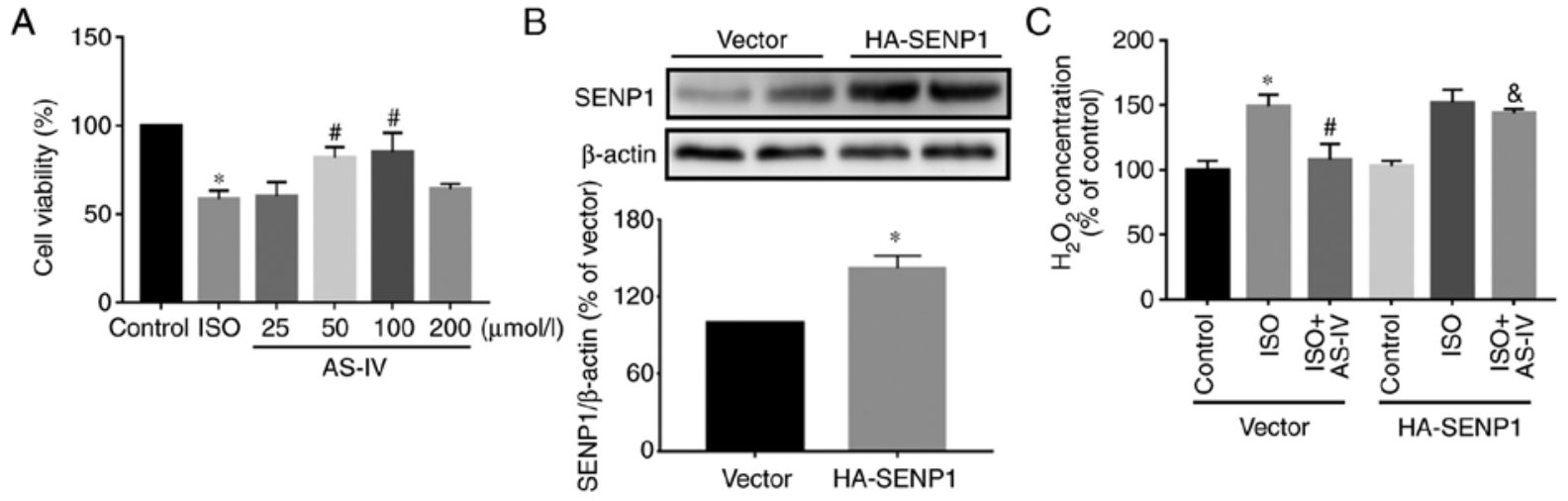

Figure 5. Effect of Senp1-overexpression on $\mathrm{H}_{2} \mathrm{O}_{2}$ in HL-1 cells. (A) The effects of different concentrations of AS-IV (25, 50, 100 or $\left.200 \mu \mathrm{mol} / \mathrm{l}\right)$ on the survival of HL-1 cells subjected to ISO. One-way ANOVA, followed by Tukey's test. (B) The expression of Senp1 protein was examined by Western blotting following HA-Senpl plasmid transfection. T-test. (C) Compared with the control (dimethyl sulfoxide, $0.1 \%$ ), $\mathrm{H}_{2} \mathrm{O}_{2}$ was increased in the $20 \mu \mathrm{mol} / 1 \mathrm{ISO}$-induced HL-1 cells. AS-IV $(50 \mu \mathrm{mol} / 1, \mathrm{n}=6)$ prevented the ISO-induced increase in $\mathrm{H}_{2} \mathrm{O}_{2}$, which was inhibited by Senpl-overexpression ( $\left.\mathrm{n}=7\right)$. Data are presented as the mean \pm standard deviation. Two-way ANOVA followed by Bonferroni's test. " $\mathrm{P}<0.05$ vs. control; ${ }^{\prime} \mathrm{P}<0.05$ vs. HF; ${ }^{2} \mathrm{P}<0.05$ vs. $\mathrm{HF}+\mathrm{AS}-\mathrm{IV}$. $\mathrm{H}_{2} \mathrm{O}_{2}$, hydrogen peroxide; AS-IV, astragaloside; ANOVA, analysis of variance; ISO, isoprenaline; Senp1, small ubiquitin-like modifier-specific protease 1.

with the HF group, AS-IV inhibited the decrease in $\Delta \psi \mathrm{m}$, while Senp1-overexpression in HL-1 cells abrogated the effect of AS-IV (Fig. 7). The TUNEL assay also demonstrated that, compared with the HF group, AS-IV decreased the apoptotic rate, while Senp1-overexpression inhibited the protective effect of AS-IV (Fig. 8).

\section{Discussion}

CHF is the end-stage of numerous cardiovascular diseases, which severely affect the life span and quality of life of patients and is a major public health problem. According to epidemiological data, there are more than 5.8 million patients with CHF in the United States and 23 million worldwide (13). It is estimated that the 5-year and 10-year survival rates of $\mathrm{CHF}$ patients are 50 and $10 \%$, respectively, and these are even lower in developing countries (14). Ventricular remodeling is an important pathological feature of $\mathrm{CHF}$, including ventricular wall thickening, ventricular dilatation and collagen fiber hyperplasia, leading to ventricular dysfunction, increased oxygen consumption and even cardiac death (15). Inhibition of ventricular remodeling is the primary therapeutic target for patients with CHF. The results of the present study demonstrated that AS-IV prevents ventricular remodeling by attenuating mitochondrial dysfunction, including the burst of ROS, the loss of $\Delta \Psi_{\mathrm{m}}$ and the release of apoptotic factors via SENP1.

The effect of astragalus in the treatment of heart failure has been verified in a variety of animal models. Its protective effects involve improving myocardial contraction, protecting myocardial cells, regulating the neuroendocrine system and inhibiting left ventricular remodeling (16). In acute HF induced by pentobarbital sodium, AS-IV increased systolic and diastolic functions without increasing myocardial oxygen consumption (17). In CHF rats induced by ligation of the left coronary artery (LAD), AS-IV significantly improved cardiovascular parameters and cardiac function (18). AS-IV 

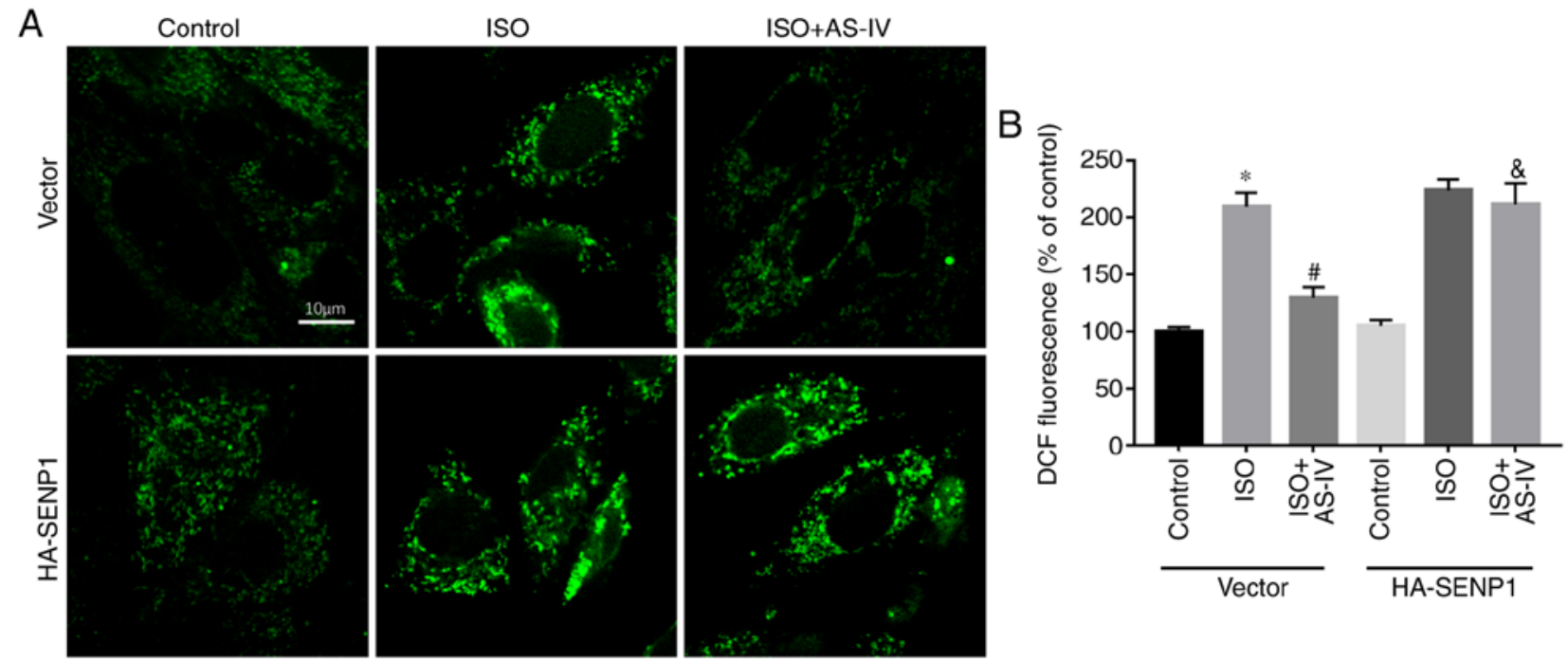

Figure 6. Effect of Senp1-overexpression on ROS generation in HL-1 cells. (A) Compared with the control (dimethyl sulfoxide, 0.1\%), DCF fluorescence was increased in $20 \mu \mathrm{mol} / 1$ ISO-induced HL-1 cells. AS-IV (50 $\mu \mathrm{mol} / 1, \mathrm{n}=6)$ prevented the ISO-induced increase in DCF fluorescence, which was inhibited by Senp1-overexpression. (B) Summarized data of DCF fluorescence $(n=7)$. Data are presented as the mean \pm standard deviation. Two-way analysis of variance, followed by Bonferroni's test. " $\mathrm{P}<0.05$ vs. control; " $\mathrm{P}<0.05$ vs. HF; ${ }^{\circledR} \mathrm{P}<0.05$ vs. HF+AS-IV. Senp1, small ubiquitin-like modifier-specific protease 1 ; ROS, reactive oxygen species; ISO, isoprenaline; AS-IV, astragaloside.

A
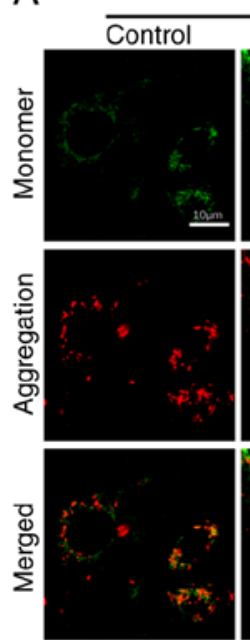

Vector
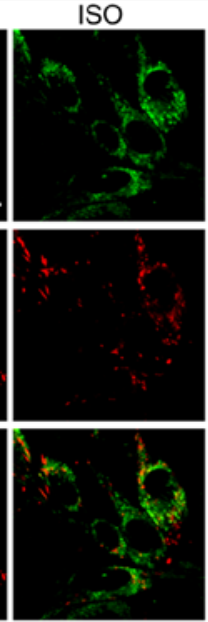
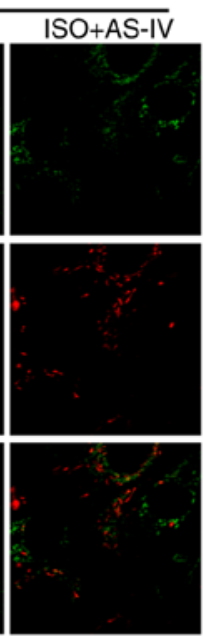

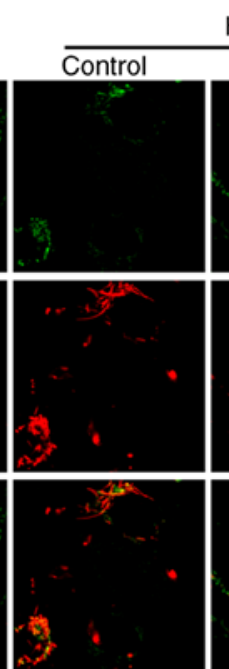

HA-SENP1
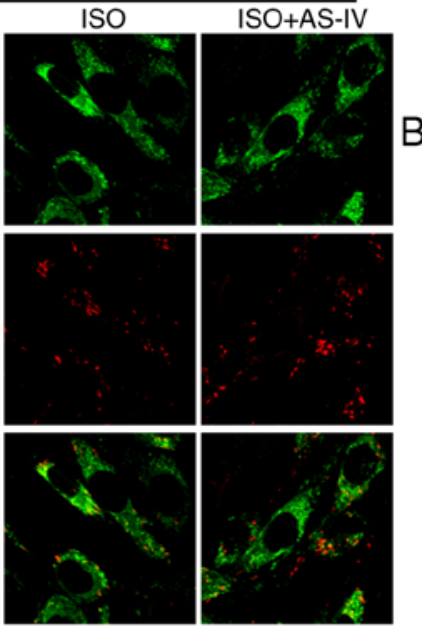

B.

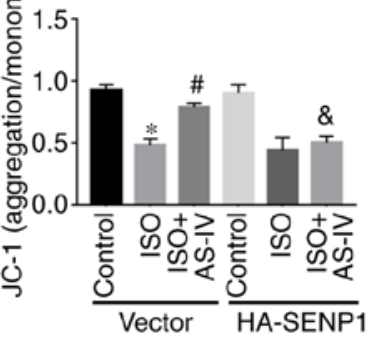

Figure 7. Effect of Senp1-overexpression on mitochondrial membrane potential in HL-1 cells. (A) Compared with the control (dimethyl sulfoxide, $0.1 \%$ ), the JC-1 ratio (aggregate/monomer) was decreased in $20 \mu \mathrm{mol} / 1 \mathrm{ISO}$-induced HL-1 cells. AS-IV (50 $\mu$ mol/1, n=7) prevented the ISO-induced decrease in the JC-1 ratio, which was inhibited by Senp1-overexpression $(n=8)$. (B) Summarized data of the JC-1 ratio. Data are presented as the mean \pm standard deviation. Two-way analysis of variance followed by Bonferroni's test. ${ }^{*} \mathrm{P}<0.05$ vs. control; ${ }^{\#} \mathrm{P}<0.05$ vs. HF; ${ }^{\text {P }}<0.05$ vs. HF+AS-IV. Senp1, small ubiquitin-like modifier-specific protease 1; ISO, isoprenaline; AS-IV, astragaloside.

treatment reversed FS, the peak values of left ventricular pressure and left ventricular systolic pressure (LVSP), and the increase in diastolic left ventricular diameter (LVIDd) and systolic left ventricular diameter (LVIDs) (18). The present study also revealed that AS-IV inhibited the increase in heart weight and heart mass/body weight ratio, the decrease in EF and FS, and the increase in LVIDd and LVIDs in mice subjected to pressure overload-driven heart failure. AS-IV reversed the morphological changes of the myocardial tissue induced by TAC. Similarly, AS-IV (50 and $100 \mu \mathrm{mol} / \mathrm{l}$ ) alleviated the HL-1 cell damage induced by ISO. However, 25 and $200 \mu \mathrm{mol} / 1 \mathrm{AS}-\mathrm{IV}$ did not demonstrate any protective effect on ISO-induced cardiomyocyte injury, suggesting that the treatment dose requires consideration, and the mechanism of AS-IV may vary between high and low doses of the drug.

Basic and clinical studies have demonstrated that ROS (superoxide, hydrogen peroxide, hydroxyl radical) produced by $\mathrm{HF}$ myocardium are increased. Excessive ROS accumulation will not only cause non-specific oxidative damage to DNA, protein and lipids, but also regulate redox-related signaling cascades, leading to further myocardial damage (19). It has been demonstrated that in $A p o E^{-/-}$mice, AS-IV treatment decreased oxidative stress, inhibited cardiac remodeling and improved ventricular function by decreasing nicotinamide adenine dinucleotide phosphate oxidase (NOX) 
A

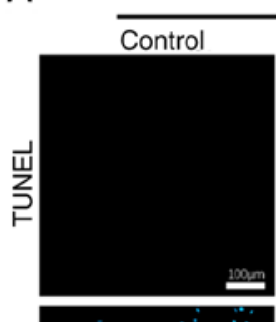

Vector

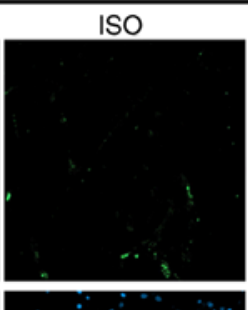

ISO
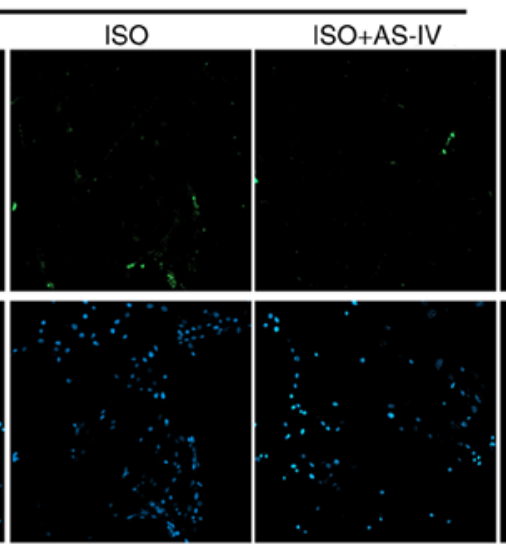

B

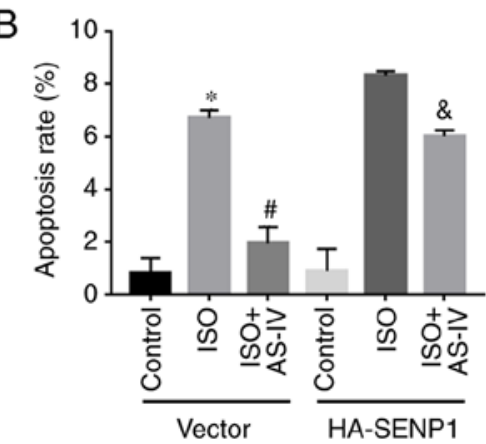

Figure 8. (A) TUNEL staining was used to examine cell apoptosis in HL-1 cells. Compared with the control (dimethyl sulfoxide, 0.1\%), cell apoptosis was increased in $20 \mu \mathrm{mol} / 1$ ISO-induced HL-1 cells. AS-IV $(50 \mu \mathrm{mol} / 1, \mathrm{n}=10)$ prevented the ISO-induced increase in cell apoptosis, which was inhibited by Senp1-overexpression. (B) Summarized data of the cell apoptotic rate $(n=7)$. Two-way analysis of variance followed by Bonferroni's test. Data are presented as the mean \pm standard deviation. ${ }^{*} \mathrm{P}<0.05$ vs. control; ${ }^{~} \mathrm{P}<0.05$ vs. HF; ${ }^{\circledR} \mathrm{P}<0.05$ vs. HF+AS-IV. ISO, isoprenaline; AS-IV, astragaloside; Senp1, small ubiquitin-like modifier-specific protease 1 .

expression and/or increasing superoxide dismutase (SOD) expression (20). Yang et al (21) have reported that AS-IV significantly enhanced cell viability, increased glutathione peroxidase and SOD activity, and decreased ROS production, thereby serving a protective role in cardiomyocytes against hypoxia/reoxygenation. Similarly, AS-IV decreased the levels of ROS and $\mathrm{H}_{2} \mathrm{O}_{2}$ and inhibited the decrease in $\Delta \psi \mathrm{m}$ in HF mice (17). It has been reported that SENP1 abnormality serves an important role in oxidative stress injury (22). Therefore, the present study further investigated whether AS-IV inhibits myocardial oxidative stress in HF mice by modulating Senp1 expression. In the present study, the expression of Senp1 in myocardial tissue samples from the different treatment groups was examined. The results showed that, compared with the control group, the expression of Senp1 protein in the HF group was increased, and this upregulation was inhibited by AS-IV treatment. Therefore, inhibition of Senpl expression may serve an important role in the improvement of ventricular function by AS-IV. To verify this hypothesis, an Senpl-overexpression plasmid was constructed and the changes in $\mathrm{ROS}, \mathrm{H}_{2} \mathrm{O}_{2}$ and $\Delta \psi \mathrm{m}$ following Senp1-overexpression in HL-1 cells were investigated. Senp1-overexpression effectively inhibited the protective effect of AS-IV against oxidative stress in the HF model. The findings of the present study preliminarily confirm the connection between SENP1 in AS-IV-induced cardio protection. However, the specific target of Senp1 and the free radical types regulated by Senp1 following AS-IV treatment remain unknown and will be the focus in future studies.
The present study demonstrated that AS-IV decreased oxidative stress and mitochondrial damage, inhibited ventricular remodeling and improved cardiac function by decreasing Senp1-upregulation in HF mice. The discovery of this mechanism provides a novel theoretical basis and clinical reference for the treatment of HF. However, the present study only investigated the role of Senp1 in AS-IV-treated cardiomyocytes; therefore, the specific target of Senp1 and the free radical types regulated by Senp1 remain to be identified in order to provide a more comprehensive theoretical basis for the protective effect of AS-IV against cardiovascular diseases.

\section{Acknowledgements}

Not applicable.

\section{Funding}

The present study was supported by grants from Tianjin Natural Science Foundation of China (grant. no. 19JCZDJC35200) and Tianjin Special Project of New Generation Artificial Intelligence Technology (grant. no. 18ZXZNSY00260).

\section{Availability of data and materials}

The datasets used and/or analyzed during the current study are available from the corresponding author on reasonable request. 


\section{Authors' contributions}

$\mathrm{JL}$ and $\mathrm{XL}$ designed the experiments. YL, XB and NX performed the experiments, and collected and analyzed the data. SD and JY drafted the manuscript. JY analyzed the data. SD interpreted data and wrote the manuscript. All authors agreed to be accountable for all aspects of the work in ensuring that questions related to the accuracy and integrity of any part of the work are appropriately investigated and resolved. XL and SD confirm the authenticity of all the raw data. All authors read and approved the final manuscript.

\section{Ethics statement and consent to participate}

The present study was performed in accordance with the Guidelines of the Ethics Committee of Tianjin Medical University Cancer Institute and Hospital (Tianjin, China). Animal experiments were approved by the Animal Ethics Committee of Tianjin Fifth Central Hospital (Tianjin, China).

\section{Patient consent for publication}

Not applicable.

\section{Competing interests}

The authors declare that they have no competing interests.

\section{References}

1. McMurray JJ and Pfeffer MA: Heart failure. Lancet 365: 1877-1889, 2005.

2. Ponikowski P, Voors AA, Anker SD, Bueno H, Cleland JGF, Coats AJS, Falk V, González-Juanatey JR, Harjola VP, Jankowska EA, et al: 2016 ESC Guidelines for the diagnosis and treatment of acute and chronic heart failure: The task force for the diagnosis and treatment of acute and chronic heart failure of the European society of cardiology (ESC)Developed with the special contribution of the heart Failure Association (HFA) of the ESC. Eur Heart J 37: 2129-2200, 2016.

3. Ishihara S, Kawakami R, Nogi M, Hirai k, Hashimoto Y, Nakada Y, Nakagawa H, Ueda T, Nishida T, Onoue K, et al: Incidence and clinical significance of 30-day and 90-day rehospitalization for heart failure among patients with acute decompensated heart failure in Japan-From the NARA-HF Study. Circ J 84: 194-202, 2020.

4. Aimo A, Januzzi JL Jr, Bayes-Genis A, Vergaro G, Sciarrone P, Passino $\mathrm{C}$ and Emdin M: Clinical and prognostic significance of SST2 in heart failure: JACC review topic of the week. J Am Coll Cardiol 74: 2193-2203, 2019.

5. Feusette P, Gierlotka M, Tukiendorf A, Płonka J, Bugajski J, Łabuz-Roszak B and Bryk R: Heart failure in opole voivodeshipepidemiology and future perspectives. Wiad Lek 72: 112-119, 2019 (In Polish)

6. Jia Q, Wang L, Zhang X, Ding Y, Li H, Yang Y, Zhang A, Li Y, Lv $S$ and Zhang J: Prevention and treatment of chronic heart failure through traditional Chinese medicine: Role of the gut microbiota. Pharmacol Res 151: 104552, 2020.
7. Zhang J, Wu C, Gao L, Du G and Qin X: Astragaloside IV derived from Astragalus membranaceus: A research review on the pharmacological effects. Adv Pharmacol 87: 89-112, 2020.

8. Swatek KN and Komander D: Ubiquitin modifications. Cell Res 26: 399-422, 2016.

9. Han ZJ, Feng YH, Gu BH, Li YM and Chen H: The post-translational modification, SUMOylation, and cancer (Review). Int J Oncol 52: 1081-1094, 2018.

10. Kunz K, Piller T and Müller S: SUMO-specific proteases and isopeptidases of the SENP family at a glance. J Cell Sci 131: jes211904, 2018.

11. Cai R, Gu J, Sun H, Liu X, Mei W, Qi Y, Xue S, Ren S, Rabinowitz JE, Wang Y, et al: Induction of SENP1 in myocardium contributes to abnormities of mitochondria and cardiomyopathy. J Mol Cell Cardiol 79: 115-122, 2015.

12. Chhunchha B, Kubo E, Singh P and Singh DP: Sumoylationdeficient Prdx6 repairs aberrant Sumoylation-mediated Sp1 dysregulation-dependent Prdx6 repression and cell injury in aging and oxidative stress. Aging (Albany NY) 10: 2284-2315, 2018.

13. Bozkurt B: What is new in heart failure management in 2017 ? Update on ACC/AHA heart failure guidelines. Curr Cardiol Rep 20: 39, 2018.

14. Kerpen K, Koutrolou-Sotiropoulou P, Zhu C, Yang J, Lyon JA, Lima FV and Stergiopoulos K : Disparities in death rates in women with peripartum cardiomyopathy between advanced and developing countries: A systematic review and meta-analysis. Arch Cardiovasc Dis 112: 187-198, 2019.

15. Frangogiannis NG: The extracellular matrix in ischemic and nonischemic heart failure. Circul Res 125: 117-146, 2019.

16. Zhang Y, Wu J, Guo S, Lin W, Zhang B, Chen X, Mo H and Zhan T: The clinical efficacy and safety of the Chinese herbal medicine Astragalus (Huangqi) preparation for the treatment of acute myocardial infarction: A systematic review of randomized controlled trials. Medicine 98: e15256, 2019.

17. Dai H, Jia G, Lu M, Liang C, Wang Y and Wang H: Astragaloside IV inhibits isoprenaline-induced cardiac fibrosis by targeting the reactive oxygen species/mitogen-activated protein kinase signaling axis. Mol Med Rep 15: 1765-1770, 2017.

18. Yuan LB, Hua CY, Gao S, Yin YL, Dai M, Meng HY, Li PP, Yang ZX and $\mathrm{Hu} \mathrm{QH}$ : Astragalus Polysaccharides attenuate monocrotaline-induced pulmonary arterial hypertension in rats. Am J Chin Med 45: 773-789, 2017.

19. van der Pol A, van Gilst WH, Voors AA and van der Meer P: Treating oxidative stress in heart failure: Past, present and future. Eur J Heart Failure 21: 425-435, 2019.

20. Song Z, Wei D, Chen Y, Chen L, Bian Y, Shen Y, Chen J and Pan Y: Association of astragaloside IV-inhibited autophagy and mineralization in vascular smooth muscle cells with lncRNA H19 and DUSP5-mediated ERK signaling. Toxicol Appl Pharmacol 364: 45-54, 2019.

21. Yang P, Zhou Y, Xia Q, Yao L and Chang X: Astragaloside IV regulates the $\mathrm{PI} 3 \mathrm{~K} / \mathrm{Akt} / \mathrm{HO}-1$ signaling pathway and inhibits H9c2 cardiomyocyte injury induced by hypoxia-reoxygenation. Biol Pharm Bull 42: 721-727, 2019.

22. Du FL, Dong WB, Zhang C, Li QP, Kang L, Lei XP, Guo L and Zhai XS: Budesonide and poractant alfa prevent bronchopulmonary dysplasia via triggering SIRT1 signaling pathway. Eur Rev Med Pharmacol Sci 23: 11032-11042, 2019.

(i) (9) This work is licensed under a Creative Commons Attribution-NonCommercial-NoDerivatives 4.0 International (CC BY-NC-ND 4.0) License. 